\title{
MODÉLISATION DE L'ÎLOT DE CHALEUR URBAIN À STRASBOURG
}

\author{
Pierre KASTENDEUCH ${ }^{1}$, Georges NAJJAR ${ }^{1}$, Pierre LACARRERE ${ }^{2}$ \\ et Jérôme COLIN ${ }^{1}$
}

${ }^{1}$ UDS/LSIIT UMR 7005 - CNRS

Faculté de Géographie et d'aménagement, 3 rue de l'Argonne,

67083 Strasbourg Cedex kasten@unistra.fr

${ }^{2}$ CNRM/GAME URA 1357 - CNRS

42 Avenue Gaspard Coriolis, 31057 Toulouse Cedex 01

\begin{abstract}
Résumé
Les modèles météorologiques qui travaillent à méso-échelle intègrent de nouveaux schémas de surface qui ont été spécialement conçus pour simuler le comportement des villes. Ces schémas les rendent en principe aptes à simuler spécifiquement les contraintes qu'exerce la ville sur les basses couches de l'atmosphère. Lorsqu'ils sont activés, ils permettent d'étudier le comportement de la couche limite urbaine ou les interactions qui existent entre la ville et la campagne. Cependant, avant d'accorder une confiance aveugle à ces modèles, il est indispensable d'en vérifier les aptitudes. C'est pourquoi, dans un premier temps, il est nécessaire de faire la simulation d'un cas réel pour lequel on dispose de données observées servant à valider la simulation. L'objectif de cet article consiste à présenter un certain nombre d'analyses qui ont été réalisées à partir d'une simulation dont les résultats ont été validés et vérifiés par une confrontation avec des données de terrain. Cette simulation concerne l'agglomération de Strasbourg (450 000 habitants) et a pour objectif d'en reproduire l'îlot de chaleur urbain. Elle a été effectuée à l'aide de la version standard du modèle Méso-NH, qui est un modèle qui appartient conjointement à Météo-France et au Laboratoire d'Aérologie de l'Université Paul Sabatier de Toulouse. La simulation a été réalisée pour des journées anticycloniques d'été pour lesquelles les conditions météorologiques sont favorables à l'apparition du phénomène : à savoir un ensoleillement maximum et des vitesses de vent faibles. La simulation se prolonge sur 5 jours, du 13 au 17 août 2002. Le 13 août est encore un peu perturbé et est marqué par des passages nuageux. C'est une journée d'initialisation et de mise à l'équilibre des champs du modèle. La procédure de simulation consiste à initialiser les champs du modèle et à les contraindre périodiquement aux limites (toutes les 6h) grâce aux réanalyses du centre de prévision européen (CEPMMT), et ceci afin d'en limiter la dérive. La qualité de la simulation a été vérifiée à l'aide de données qui ont été acquises en 2002 lors d'une campagne de mesures réalisée dans le cadre d'un projet de recherche appelé Rayonnement Energie dans la Couche Limite Urbaine à Strasbourg (RECLUS). Les résultats ont été comparés aux mesures pour un certain nombre de variables fondamentales et dans l'ensemble, force est de constater que pour la plupart des paramètres analysés, les résultats obtenus sont très cohérents. Par conséquent, il est possible de se baser sur cette simulation pour réaliser des investigations que ne permettent pas (ou difficilement) les réseaux de mesures (comme par exemple l'obtention d'informations spatialisées). Après une journée de simulation, lorsque le beau temps s'installe vraiment (i.e. le 14 août), l'îlot de chaleur apparaît systématiquement les nuits sur Strasbourg. La simulation est alors utilisée pour réaliser une analyse fine des processus et de leurs conséquences sur l'atmosphère urbaine. En conclusion, cette étude confirme l'utilité de ces modélisations du climat urbain qui sont maintenant suffisamment fiables pour devenir indispensables en recherche fondamentale (pour mettre en évidence les mécanismes), mais aussi en recherche appliquée (dans la perspective de l'aménagement urbain).
\end{abstract}

Mots-clés : îlot de chaleur, modélisation, Méso-NH, bilan d'énergie.

\section{Abstract}

\section{Simulation of the urban heat island at Strasbourg}

The mesoscale meteorological models include new surface schemes that have been design to simulate the urban behaviour. These schemes made them able to simulate the constraint exerted by the town on the low level layers of the atmosphere. When they are activated, they allow to study the behaviour of the urban boundary layer dynamics or the interaction that exists between the town and the rural areas. But, all these models have their own weaknesses and it is necessary to verify the quality of their results. It is the reason why, in a first step, it is necessary to do a simulation on a real case, for which validation data are available. The aim of this paper is to

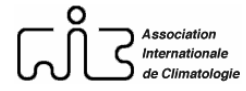


present a simulation of the urban heat island at Strasbourg (450 000 inhabitants). The simulation is done with the Méso-NH model of Météo-France and the Laboratoire d'Aérologie of the Paul Sabatier University (Toulouse). It is done for anticyclonic summer days for which the weather conditions permits the development of the phenomenon (low winds and maximum incoming radiation). The simulation extends on 5 days (from august 13 to 17,2002 ). The first day (august 13) was still concerned by some clouds and is considered as an initialisation day. The simulation procedure consists to initialise the meteorological fields and to constrain them every 6 hours with the reanalysis of the European Prevision Centre (CEPMMT). The quality of the simulation was verified with data acquired during a measurement campaign that took place in 2002 in the frame of a research project called (RECLUS). The simulation results were compared to the measurements for some fundamental variables. On the whole, the results are satisfactory. Consequently, it is possible to use the simulation to investigate the urban climate better than with the measurements alone (especially with regard to the spatial information). After one simulation day, when the weather conditions become ideal (the august 14), the urban heat island is fully developed during all the nights. The simulation can be used to do an analysis of the processes and their consequences on the urban atmosphere. As a conclusion, this study confirms the usefulness to obtain realistic simulations of the urban climate. The results are helpful in fundamental research (to understand the mechanisms) and also in the field of the applied research (especially for the need of urban planning).

Keywords: heat island, simulation, Meso-NH, heat budget.

\section{Introduction}

De par l'étendue de leurs surfaces artificielles, et sous certaines conditions météorologiques, les villes sont capables de transformer significativement les caractéristiques des basses couches de l'atmosphère avec lesquelles elles sont en contact. Elles donnent alors naissance à un climat assez différent de celui des zones rurales environnantes (Heino, 1979 ; Landsberg, 1981; Oke 1987). Les spécialistes de climatologie urbaine distinguent actuellement plusieurs tranches d'atmosphère. Entre le niveau moyen des toits et le sol, ils identifient une couche d'air connue sous le nom de canopée urbaine (CU). La CU est constituée essentiellement par les volumes d'air qui occupent les rues. C'est donc une couche extrêmement fractionnée par la présence des bâtiments, et lorsque le bâti devient vraiment très dense et continu (bâtiments jointifs) les connexions entre les volumes d'air qu'elle renferme ne s'y opèrent qu'au niveau des intersections des rues. Dans ce cas, elle ne communique avec l'atmosphère supérieure que par le sommet de la rue. La CU est la couche qui subit les plus fortes transformations climatiques, puisqu'elle est en contact étroit avec les éléments urbains et elle est influencée non seulement à sa base (par les éléments qui constituent le sol de la ville), mais aussi latéralement par les murs des bâtiments.

Au-dessus de la CU, on identifie une seconde couche, connue sous le nom de couche limite urbaine (CLU). Elle s'étend à partir du niveau moyen des toits sur une épaisseur très variable en fonction des conditions météorologiques. La CLU est influencée à sa base de deux façons : i) par l'air qui s'échappe par le sommet des rues et qui provient de la CU sous-jacente ; ii) par le fait qu'elle se trouve en contact avec la partie supérieure des éléments architecturaux (qui sont à ce niveau essentiellement formés de surfaces de toitures). Toutes ces influences vont se mélanger au sein de la CLU. Cette dernière peut prendre la forme d'un dôme par vent faible $\left(<3 \mathrm{~m} . \mathrm{s}^{-1}\right)$ ou d'un panache urbain par vent un peu plus fort. Mais, au-delà d'une certaine vitesse de vent (dont le seuil varie en fonction de la taille de la ville), l'advection devient suffisamment prépondérante pour empêcher toute individualisation de l'atmosphère urbaine.

Lorsque toutes les conditions sont réunies, la conséquence la plus connue de ces processus est l'apparition du fameux îlot de chaleur urbain nocturne (ICU). Ce type de conditions météorologiques favorise également l'augmentation de la concentration en polluants dans l'air, puisque les vents faibles sont aussi peu favorables à leur dispersion et que le rayonnement diurne important favorise les réactions photochimiques. 
Cet article présente une tentative de simulation des basses couches de l'atmosphère urbaine à l'aide d'un modèle numérique, pour un épisode météorologique qui a favorisé l'apparition de l'îlot de chaleur nocturne. C'est l'agglomération de Strasbourg, située dans le nord-est de la France (région Alsace) qui sert de terrain d'expérimentation. L'ICU est étudié sous deux angles : son évolution temporelle à court terme (quelques jours) et son organisation spatiale (horizontale et verticale). La simulation a été effectuée à l'aide du modèle Méso-NH dont le code a été développé par le CNRM (Météo-France) et le Laboratoire d'Aérologie de l'Université Paul Sabatier à Toulouse.

\section{Présentation du domaine et des moyens d'étude}

L'agglomération de Strasbourg compte 450000 habitants répartis sur $306 \mathrm{~km}^{2}$ (données de 1999). Elle est située en bordure d'un fleuve (le Rhin) qui marque la frontière entre la France et l'Allemagne. On note une topographie régionale assez particulière, puisque le Rhin coule au milieu d'un fossé tectonique qui fait environ $50 \mathrm{~km}$ de large dont les bordures à l'est et à l'ouest sont constituées par les deux chaînes de moyenne montagne que sont la Forêt-Noire (côté allemand) et les Vosges (côté français).

Des conditions météorologiques bien précises ont été choisies pour étudier l'impact de la ville de Strasbourg sur les basses couches de l'atmosphère. Ce sont des journées d'été, anticycloniques, avec beau temps, un ensoleillement maximum et un vent synoptique faible. En effet, ces conditions estivales facilitent l'apparition de l'îlot de chaleur puisque les transferts d'énergie locaux l'emportent face à l'advection.

Les phénomènes décrits dans cet article se déroulent au cours d'une série de journées qui s'étalent du 13 au 19 août 2002. La situation météorologique de ces sept jours n'a rien d'exceptionnel. Un anticyclone centré sur le nord de la France a permis de dévier momentanément la trajectoire des perturbations sur l'Europe du Nord (figure 1).

Figure 1 : Situation météorologique au 14 août 2002 à 0 h d'après l'analyse de surface de l'UKMO (United Kingdom Meteorological Office). Meteorological weather map of august 14, 2002 at 0 UTC, after UKMO.

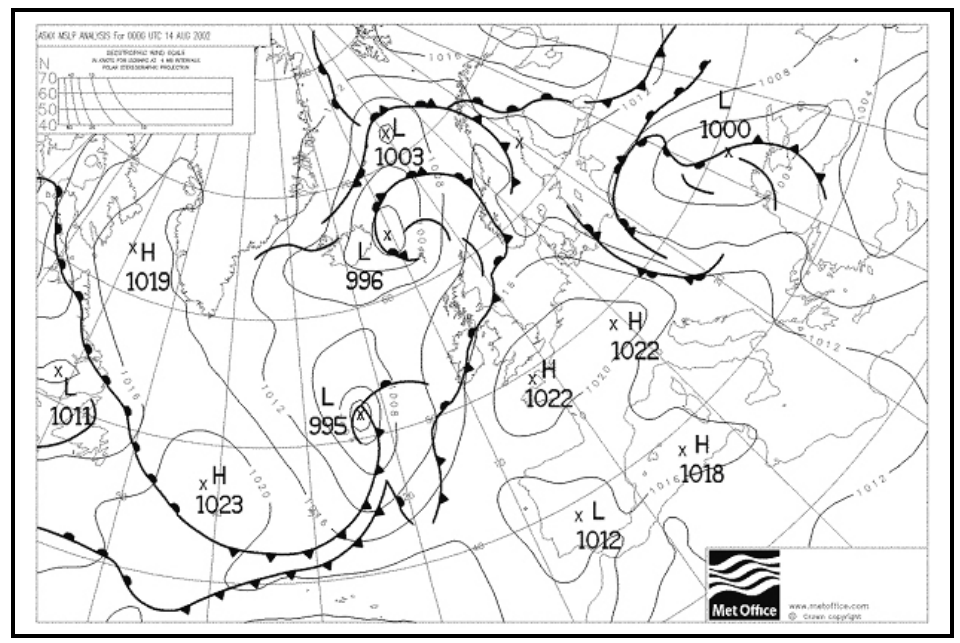

La première journée de la série (celle du 13 août) fait suite à des passages perturbés, pluvieux, qui ont humidifié le sol. Le 13, le vent est encore à l'ouest, mais le ciel légèrement nuageux ne génère plus de pluie. Dans la nuit du 13 au 14 août, la situation météorologique évolue avec l'arrivée de l'anticyclone. Le vent change de direction et s'établit progressivement au nord-est, canalisé par le fossé rhénan, ce qui est courant dans ce cas. Les températures maximales ainsi que l'amplitude thermique diurne augmentent grâce à une faible nébulosité. Ainsi, c'est véritablement dans la nuit du 14 au 15 août que les phénomènes locaux s'affermissent et que l'îlot de chaleur urbain se développe pleinement. Les jours qui

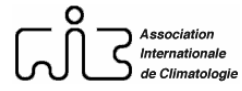


suivent sont tous très ensoleillés et chauds et offrent des conditions idéales pour étudier la couche limite urbaine et son évolution.

Ces journées ont aussi l'avantage de faire partie d'une période d'observations intensive (POI) qui s'est déroulée dans le cadre du projet de recherche Rayonnement Energie dans la Couche Limite Urbaine à Strasbourg (RECLUS ; Najjar et al., 2004). Les données acquises durant cette POI ont été mises à contribution pour prouver la qualité des simulations (Kastendeuch et Najjar, 2009a), ce qui permet de présenter dans cet article des analyses sur les mécanismes urbains.

La modélisation a été effectuée en collaboration avec le Centre National de la Recherche Météorologique (CNRM, Météo-France) en utilisant le modèle de recherche météorologique Méso-NH. Ce modèle numérique est actuellement considéré comme l'un des plus aboutis de sa catégorie puisque, en tant que modèle de recherche, il intègre en permanence les derniers raffinements en matière de modélisation à méso-échelle. Pour les besoins de la simulation envisagée, les schémas de surface TEB pour la ville (Masson, 2000) et ISBA pour la campagne (Noilhan et Planton, 1989 ; Noilhan et Mahfouf, 1996) ont été activés.

Ces schémas de surface sont des sous-programmes du modèle atmosphérique (Martilli et al., 2002 ; Zhang et al., 2008). Ils ont pour objectif principal de simuler le comportement des différents types d'occupation du sol et de calculer les échanges de chaleur, de masse et de mouvement qui se produisent entre le sol et l'atmosphère. Il en existe plusieurs dans Méso$\mathrm{NH}$, avec un pour chaque grand type d'occupation du sol (ville, campagne, eau). Le schéma de surface qui s'occupe de la ville se nomme TEB (pour Town Energy Budget). Il permet de simuler de manière aussi réaliste que possible le comportement spécifique des zones urbaines et notamment, tout se qui se déroule au sein de la CU. Notons d'ailleurs que Méso-NH (comme tous les modèles météorologiques) travaille à partir d'un maillage atmosphérique qui possède une certaine résolution horizontale et verticale (puisque l'atmosphère y est découpée en boites). La résolution horizontale au sol dépend de la simulation qui est effectuée, mais elle peut varier de quelques kilomètres à quelques mètres carrés. Aussi, l'occupation du sol à l'intérieur de ces mailles est forcément disparate. Chaque maille est considérée comme un patchwork de plusieurs types d'occupation du sol, dont il faut définir les proportions (pourcentage de ville, forêts, eau, prairie...). Ainsi, pour la zone urbaine de Strasbourg, on considère que les mailles se composent à $90 \%$ de surfaces bâties (routes, bâtiments...) et pour $10 \%$ de végétation (chiffres obtenus après une analyse sommaire de photographies aériennes). C'est pourquoi plusieurs schémas de surface fonctionnent simultanément pour chaque maille, chacun pour simuler le comportement spécifique d'un type d'occupation du sol. Les résultats sont ensuite pondérés en fonction des proportions, pour obtenir le comportement moyen de la maille.

Pour fonctionner correctement, ces schémas de surface ont besoin de connaître les caractéristiques fines de l'occupation du sol qu'ils sont chargés de simuler. C'est aussi le cas de TEB pour la ville, pour lequel les zones artificielles sont décrites à l'aide d'un certain nombre de paramètres, comme par exemple une hauteur moyenne des bâtiments, une largeur moyenne des rues, l'épaisseur et le type de matériaux (via des coefficients d'émissivité, de conductivité thermique)... Ces paramètres possèdent des valeurs par défaut qui ont été conservées pour l'essentiel, sauf pour la hauteur des bâtiments $(30 \mathrm{~m})$ et les flux anthropiques de vapeur d'eau et de chaleur sensible (émissions dues au trafic ou aux diverses activités humaines) qui ont été désactivés car ils sont difficiles à estimer.

L'un des avantages de TEB, par rapport à des schémas de surface urbains plus anciens, réside dans le fait qu'il a été le premier à calculer non pas une, mais plusieurs températures de surface : $i$ ) une pour les toitures ; ii) une pour les façades ; iii) une pour les routes. Ces 
températures lui permettent de déduire la contribution de ces différents types de surfaces aux flux de chaleur urbains. Pour une description détaillée de TEB (ou d'ISBA), il est toujours possible de se référer aux articles spécifiques qui leur sont consacrés (Noilhan et Planton, 1989 ; Noilhan et Mahfouf, 1996 ; Masson, 2000). Notons qu'actuellement, des schémas de surface encore plus sophistiqués font leur apparition, qui sont capables de travailler en vraie 3D (voir par exemple Kastendeuch et al., 2006 ; Kastendeuch et Najjar, 2009b).

Afin d'assurer le suivi temporel de la CLU avec la simulation, cette dernière s'étend sur l'ensemble des 5 jours choisis. Elle démarre à partir du 13 août à $0 \mathrm{~h}$ TU. La première journée de simulation sert surtout à initialiser et à «équilibrer » les champs du modèle. Pour obtenir les meilleurs résultats possibles, trois modèles vont travailler simultanément à trois échelles et avec trois résolutions horizontales différentes. Ces modèles emboîtés s'influencent respectivement aux limites de leurs domaines respectifs. Cette technique, connue en anglais sous le nom de "grid-nesting», permet d'assurer les interactions et le passage entre les échelles. Un premier modèle (le modèle père), d'une résolution horizontale de $10 \mathrm{~km}$, effectue une simulation météorologique à l'échelle synoptique avec une matrice de $90 * 90$ points centrée sur Strasbourg $\left(48,59^{\circ} \mathrm{N} ; 7,75^{\circ} \mathrm{E}\right)$, soit un domaine de $900^{*} 900 \mathrm{~km}$ (figure 2).

Figure 2 : Domaine de simulation du premier modèle (le modèle père). Il s'agit ici du relief. La résolution horizontale est de $10 \mathrm{~km}$, la matrice est de $90 * 90$ points, centrée sur Strasbourg $\left(48,59^{\circ} \mathrm{N} ; 7,75^{\circ} \mathrm{E}\right)$, soit un domaine de $900 * 900 \mathrm{~km}$. Simulation domain of the first model (father model). Topographic map of $10 \mathrm{~km}$ resolution, $90^{*} 90$ points, centred over Strasbourg $\left(48.59^{\circ} \mathrm{N} ; 7.75^{\circ} \mathrm{E}\right)$.

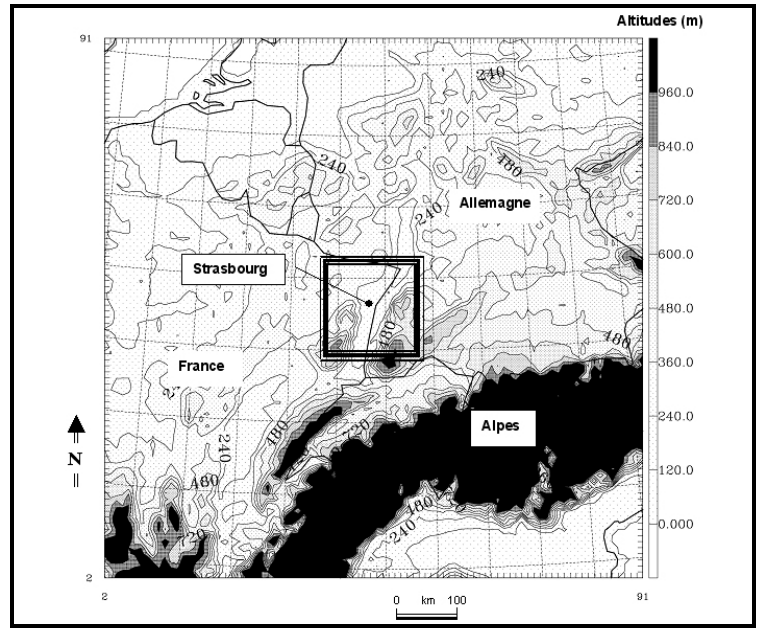

Les conditions aux limites de ce modèle sont fournies toutes les $6 \mathrm{~h}$ par les champs issus des réanalyses du CEPMMT (Centre Européen pour les Prévisions Météorologiques à Moyen Terme). Ce couplage lui permet de «coller» au plus près de la situation synoptique. Un deuxième modèle (modèle fils), d'une résolution horizontale de $2 \mathrm{~km}$, simule à l'échelle mésoclimatique avec une matrice de $80 * 80$ points centrée sur Strasbourg, soit un domaine de $160 * 160 \mathrm{~km}$ (figure 3).

Figure 3 : Domaine de simulation du second modèle (premier modèle fils). Il s'agit ici du relief. La résolution horizontale est de $2 \mathrm{~km}$, la matrice est de $80 * 80$ points, centrée sur Strasbourg $\left(48,59^{\circ} \mathrm{N}\right.$; $\left.7,75^{\circ} \mathrm{E}\right)$, soit un domaine de $160 * 160 \mathrm{~km}$. Simulation domain of the second model (first son model). Topographic map of $2 \mathrm{~km}$ resolution, $80 * 80$ points, centred over Strasbourg $\left(48.59^{\circ} \mathrm{N} ; 7.75^{\circ} \mathrm{E}\right)$.

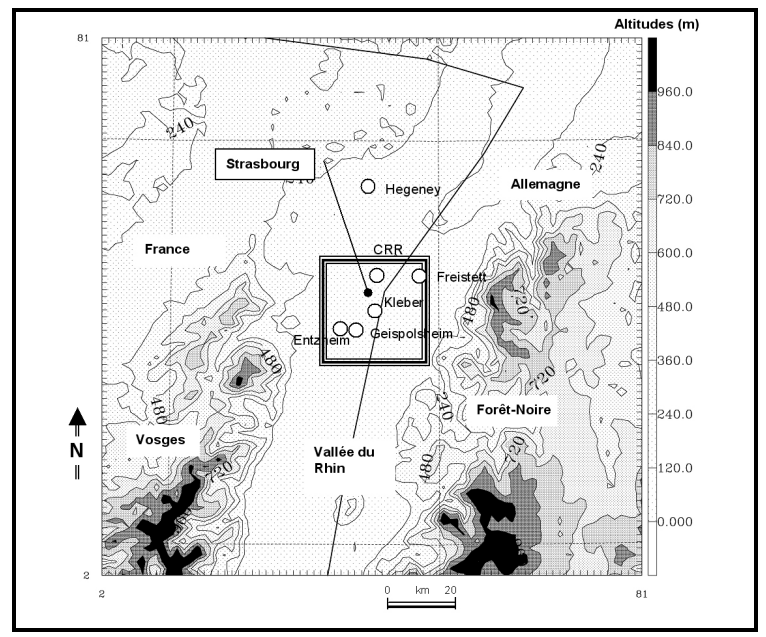


Ce modèle fils englobe le fossé rhénan. Enfin, un troisième modèle, d'une résolution horizontale de $250 \mathrm{~m}$, fournit les résultats sur la ville avec une matrice de $120 * 120$ points, centrée sur Strasbourg, soit un domaine de $30 * 30 \mathrm{~km}$ (figure 4). Ces trois modèles possèdent la même résolution verticale : 67 niveaux avec une résolution verticale de $20 \mathrm{~m}$ pour le niveau le plus proche du sol qui va en augmentant en altitude («stretching »).

Figure 4: Domaine de simulation du troisième modèle (second modèle fils). Il s'agit ici de la représentation de la fraction de zone urbaine (la ville apparaît en noir). La résolution horizontale est de $250 \mathrm{~m}$, la matrice est de $120^{*} 120$ points, centrée sur Strasbourg $\left(48,59^{\circ} \mathrm{N} ; 7,75^{\circ} \mathrm{E}\right)$, soit un domaine de $30 * 30 \mathrm{~km}$. Simulation domain of the third model (second son model). The map represents the urban fraction. $250 \mathrm{~m}$ of horizontal resolution, $120 * 120$ points, centred over Strasbourg $\left(48.59^{\circ} \mathrm{N} ; 7.75^{\circ} \mathrm{E}\right)$.

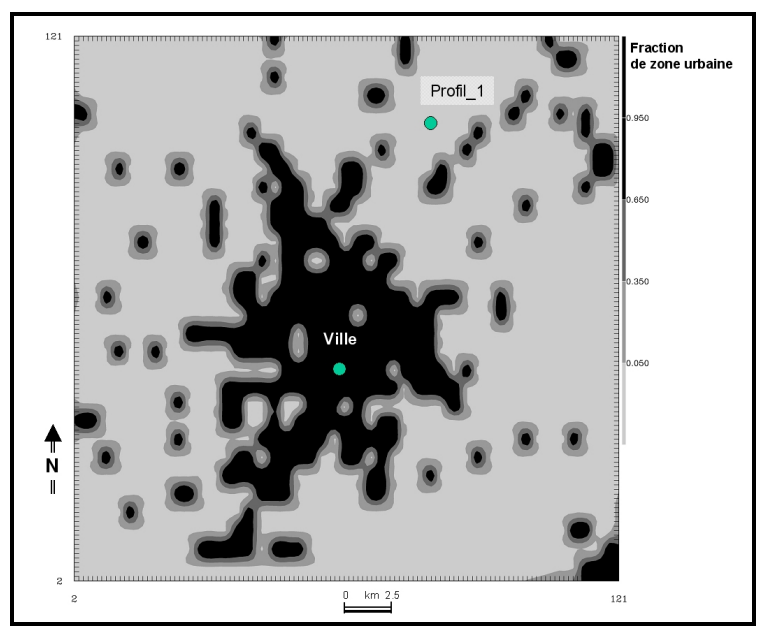

Etant donné la configuration topographique des domaines de simulation, on peut considérer que le relief va jouer un rôle non négligeable, surtout dans les deux premiers modèles. Le modèle $\mathrm{N}^{\circ} 1$ contient une partie des Alpes, et le modèle $\mathrm{N}^{\circ} 2$ englobe les massifs qui bordent le Fossé Rhénan (Vosges et Forêt-Noire). Par contre, le modèle $\mathrm{N}^{\circ} 3$ renferme peu de dénivelés puisqu'il cible la ville de Strasbourg qui est quasiment plane. C'est un avantage certain pour les phénomènes urbains que nous cherchons à simuler. En effet, lorsque la topographie est marquée, les processus de surface sont complètement perturbés par l'exposition et la pente des versants, ce qui complique l'analyse. Non seulement les flux sont influencés, mais des brises thermiques locales peuvent aussi se développer (Kastendeuch et al., 2000). Si Strasbourg semble a priori à l'écart de ce genre de problème, rien n'exclut une influence possible des reliefs proches.

Afin de prouver la qualité de la simulation, les résultats de cette modélisation ont été validés à l'aide de données qui ont été acquises en 2002, lors d'une campagne de mesures réalisée dans le cadre du projet RECLUS (cf. ci-dessus). Les résultats ont été comparés aux mesures pour un grand nombre de variables au sol et en altitude (Kastendeuch et Najjar 2009a). Dans l'ensemble, force est de constater que pour la plupart des paramètres analysés, les résultats obtenus sont excellents. Cet article n'a pas pour vocation de présenter ces validations qui sont de la même qualité que celles obtenues par d'autres schémas de surface pourtant plus récents (par exemple : Otte et al., 2004 ; Dupont et Mestayer, 2006 ; Hamdi et Schayes, 2007). Au contraire, la simulation va être utilisée pour décortiquer les processus à l'œuvre dans le climat urbain en se substituant avantageusement aux mesures.

\section{Processus radiatifs}

Les processus que nous cherchons à simuler (et notamment l'ICU) ne se développent convenablement que si la quantité de rayonnement solaire reçue au sol (le rayonnement global) est maximale durant la journée. La figure 5 retrace les diverses composantes du rayonnement net qui ont été simulées sur les cinq jours de l'expérience et notamment la quantité de rayonnement global. Les journées des 14,15 et 16 août remplissent parfaitement le critère. Le rayonnement global y atteint la valeur maximale de $860 \mathrm{~W} \mathrm{~m}^{-2}$ vers $12 \mathrm{~h}$ TU et 
son tracé suit une courbe régulière qui ne révèle aucun passage nuageux tout au long des trois jours (conformément à l'observation). En revanche, les journées des 13 et 17 août sont marquées par des passages nuageux. Ceux-ci sont très réalistes le 13, beaucoup moins le 17. Comme cette surestimation de la nébulosité au cinquième jour de la simulation a des conséquences en chaîne importantes sur la valeur de tous les autres paramètres calculés, la simulation a été arrêtée.

Figure 5 : Composantes du rayonnement net simulées pour la ville du 13 au 17 août 2002. Rayonnement global (Global), rayonnement solaire réfléchi (R), infrarouge atmosphérique (IRA) et infrarouge terrestre (IRT). Components of the net radiation simulated for the town from august 13 to august 17, 2002. Global radiation (Global), reflected solar radiation $(R)$, atmospheric infrared radiation (IRA) and terrestrial infrared radiation (IRT).

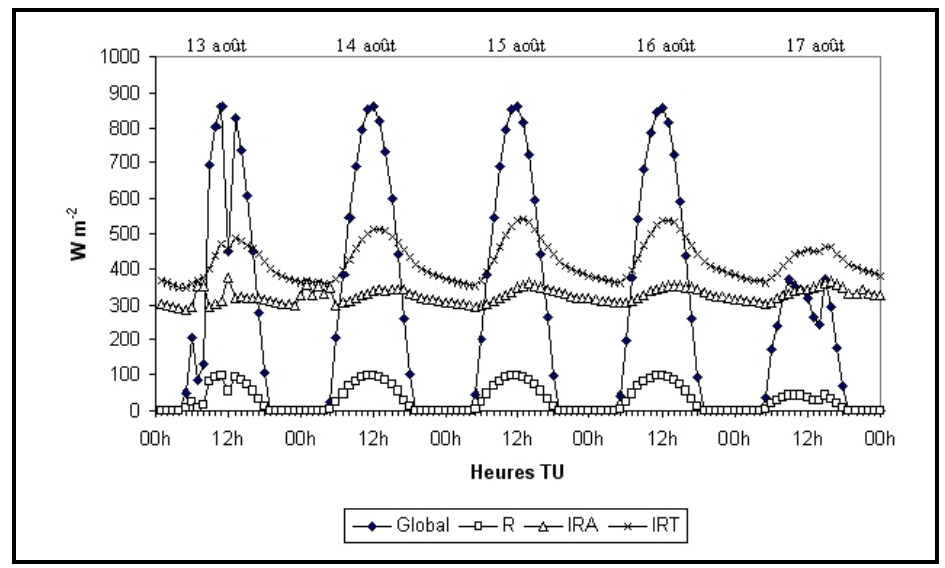

Le rayonnement global est partiellement réfléchi par les éléments qui composent le terrain urbain. Plus le rayonnement solaire réfléchi est important, moins il y a d'énergie disponible pour la surface. Etant donné les caractéristiques des matériaux introduites dans le modèle et la géométrie urbaine (notamment le rapport $\mathrm{H} / \mathrm{W}$ ), la réflexion atteint au mieux $100 \mathrm{~W} \mathrm{~m}^{-2}$ vers $12 \mathrm{~h}$ TU (cf. figure 5). Connaissant le rayonnement global incident et sa part réfléchie, cela donne un albédo moyen de 0,104 pour la maille. Ce résultat démontre que l'essentiel du rayonnement global reste bien piégé par les éléments qui composent la canopée urbaine. Cette dernière va pouvoir emmagasiner cette énergie pour se réchauffer ou la restituer sous une forme ou une autre (rayonnement infrarouge ou flux de chaleur sensible).

$\mathrm{Au}$ rayonnement global incident, il faut encore rajouter le rayonnement infrarouge en provenance de l'atmosphère. Ce terme révèle deux comportements : $i$ ) une petite oscillation journalière de l'ordre de $50 \mathrm{~W} \mathrm{~m}^{-2}$ (entre $300 \mathrm{~W} \mathrm{~m}^{-2}$ la nuit et $350 \mathrm{~W} \mathrm{~m}^{-2}$ le jour). Cette oscillation suit le cycle thermique nycthéméral de l'atmosphère; ii) une légère tendance positive qui prouve que l'atmosphère se réchauffe lentement au fil des jours.

Le rayonnement infrarouge terrestre représente quant à lui, une perte d'énergie non négligeable pour la ville. Cette composante radiative subit aussi une fluctuation journalière, en liaison avec le réchauffement et le refroidissement des éléments urbains ainsi qu'une tendance à la hausse (mais beaucoup plus prononcée que pour le rayonnement infrarouge atmosphérique), puisque sa valeur passe de 480 à $540 \mathrm{~W} \mathrm{~m}^{-2}$. Cette tendance est le fruit de l'évolution des températures de surface qui augmentent avec l'accroissement de la chaleur stockée dans les objets, ce qui prouve le réchauffement de la ville.

Le rayonnement net exprime le bilan des échanges radiatifs dans l'infrarouge et le visible. En général, ce terme pilote en grande partie le bilan d'énergie d'un objet (qui détermine luimême l'équilibre énergétique de celui-ci). Sur la figure 6, le rayonnement net de la ville est très largement positif en journée $\left(570 \mathrm{~W} \mathrm{~m}^{-2}\right)$ et légèrement négatif la nuit (entre -80 et -50 $\mathrm{W} \mathrm{m}^{-2}$ ). Ces résultats traduisent, sans grande surprise, l'importance que prend le rayonnement global lorsqu'il est actif le jour. Sa contribution explique les fortes valeurs positives diurnes (plus de rayonnement reçu que perdu). La nuit, en revanche, lorsqu'il n'y a plus que les échanges dans l'infrarouge, le rayonnement terrestre domine par rapport au rayonnement

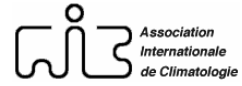


atmosphérique et le rayonnement net devient faiblement négatif (plus de rayonnement perdu que reçu).

Figure 6: Composantes du bilan d'énergie simulé pour la maille "Ville" du 14 au 16 août 2002. Rayonnement net (RN), flux de chaleur sensible $(\mathrm{H})$, flux de chaleur latente (LE) et flux de chaleur dans le sol (G). Energy balance components simulated for the town from august 14 to august 17, 2002. Net radiation (RN), sensible heat flux $(H)$, latent heat flux (LE) and ground heat flux $(G)$.

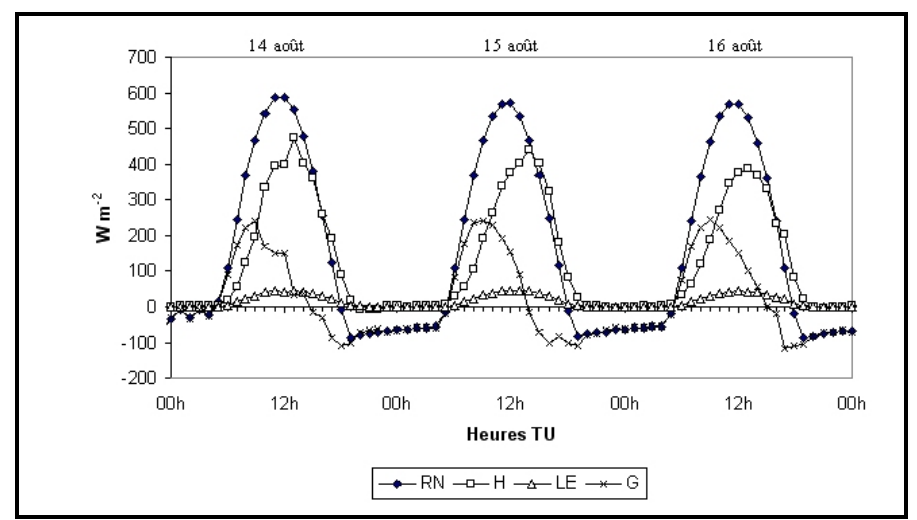

En regardant plus attentivement les résultats, on constate que la valeur du maximum du rayonnement net a tendance à diminuer insensiblement au fil des jours. La figure 7 montre bien ce phénomène et permet aussi de constater que cela tient au bilan du rayonnement infrarouge. En effet, avec le réchauffement de la ville, l'infrarouge terrestre se renforce d'avantage que l'infrarouge atmosphérique, ce qui conduit à un bilan de l'infrarouge de plus en plus négatif.

Figure 7: Rayonnement net et bilan de l'infrarouge simulés pour la maille "Ville" du 13 au 17 août 2002. Rayonnement net (RN), bilan de l'infrarouge (BIR). Net radiation and infrared radiation balance simulated for the town from august 13 to august 17, 2002. Net radiation (RN), infrared radiation balance (BIR).

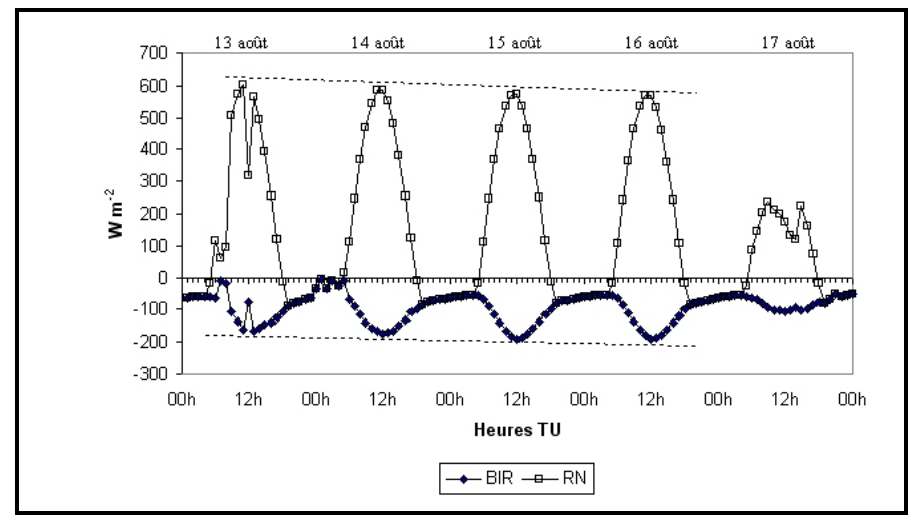

\section{Les flux de chaleur}

On désigne par flux de chaleur, les transferts d'énergie qui se produisent sous forme non radiative et qui complètent le rayonnement net dans l'établissement du bilan d'énergie d'un objet. Ils sont au nombre de trois : le flux de chaleur sensible, le flux de chaleur latente et le flux de chaleur dans le sol.

Le flux de chaleur sensible correspond au flux de chaleur échangé par conduction entre le sol et l'atmosphère. Il est très élevé en journée (figure 6) et peut atteindre des valeurs de 475 $\mathrm{W} \mathrm{m}^{-2}$. Le maximum se produit vers 13 ou $14 \mathrm{~h}$ (soit une à deux heures après le maximum du rayonnement net). L'énergie perdue par les éléments urbains par cette voie est quasiment du même ordre de grandeur que celle qui est perdue sous forme de rayonnement infrarouge. La différence fondamentale est que cette énergie sert à réchauffer directement les basses couches de l'atmosphère.

Le flux de chaleur latente est la quantité de chaleur transférée du sol vers l'atmosphère lors du changement de phase de l'eau. Pour Strasbourg, ce flux est assez faible (mais pas nul), étant donné la rareté de la végétation en ville (10\% de l'espace urbain). Le flux de chaleur latente ne dépasse pas, au mieux, $40 \mathrm{~W} \mathrm{~m}^{-2}$ (cf. figure 6). Non seulement ce mode de transfert 
de chaleur n'est pas prépondérant, mais la ville apparaît aussi comme une piètre source de vapeur d'eau pour l'atmosphère.

Le flux de chaleur dans le sol est le flux de chaleur qui est transmis par conduction au sein des objets. Dans TEB, cette variable n'est pas simulée directement, mais est obtenue en équilibrant les termes du bilan d'énergie. En conséquence, elle cumule toutes les erreurs qui ont pu être commises sur l'estimation des autres flux. C'est pourquoi, malgré la confiance que l'on peut accorder aux résultats, ce qui suit est à prendre avec réserve. Le flux de chaleur dans le sol (figure 6) suit aussi une oscillation journalière non négligeable, ce qui le rapproche plus par son comportement du flux de chaleur sensible que du flux de chaleur latente. Entre le lever du soleil et 15-16h de l'après-midi, ce flux est positif, ce qui révèle un stockage de chaleur qui atteint son summum en milieu de matinée. Le reste de la journée, c'est le destockage qui prend le relais (le flux est négatif de la fin d'après-midi au lendemain matin). Or les valeurs positives correspondent à une pénétration d'énergie calorifique dans les objets qui peut atteindre des valeurs de plus de $240 \mathrm{~W} \mathrm{~m}^{-2}$, alors que les valeurs négatives correspondent à une perte d'énergie calorifique qui peut descendre sous les $-100 \mathrm{~W} \mathrm{~m}^{-2}$. Au final, c'est plutôt le stockage qui l'emporte en moyenne sur une journée (environ $+13 \mathrm{~W} \mathrm{~m}^{-2}$ ). Ce calcul prouve bien qu'il y a une accumulation de la chaleur dans la ville. En comparant ces valeurs par rapport à celles qui sont obtenues à la campagne, on s'aperçoit que le stockage en ville est environ cinq fois plus important qu'à la campagne et que le destockage y est seulement deux fois plus important. Au final, au cours d'une journée, la ville serait capable de stocker environ 2,5 fois plus d'énergie que la campagne.

Le gros point fort de la modélisation est qu'elle permet de passer facilement d'une vision ponctuelle des phénomènes à une représentation spatiale (via des cartographies, des coupes et des transects). La carte du flux de chaleur nocturne dans le sol établie pour le 16 août à $23 \mathrm{~h}$, révèle l'organisation spatiale de ce flux (figure 8). La spécificité de la ville y apparaît clairement : c'est l'endroit où le destockage nocturne y est le plus important.

Figure 8 : Carte du flux de chaleur dans le sol (G) pour une résolution de $250 \mathrm{~m}$ (modèle $\mathrm{N}^{\circ} 3$ ) le 16 août 2002 à 23h. Map of the ground heat flux $(G)$ of august 16, 2002 at 23 UTC. The horizontal resolution is of $250 \mathrm{~m}$ (model $n^{\circ} 3$ ).

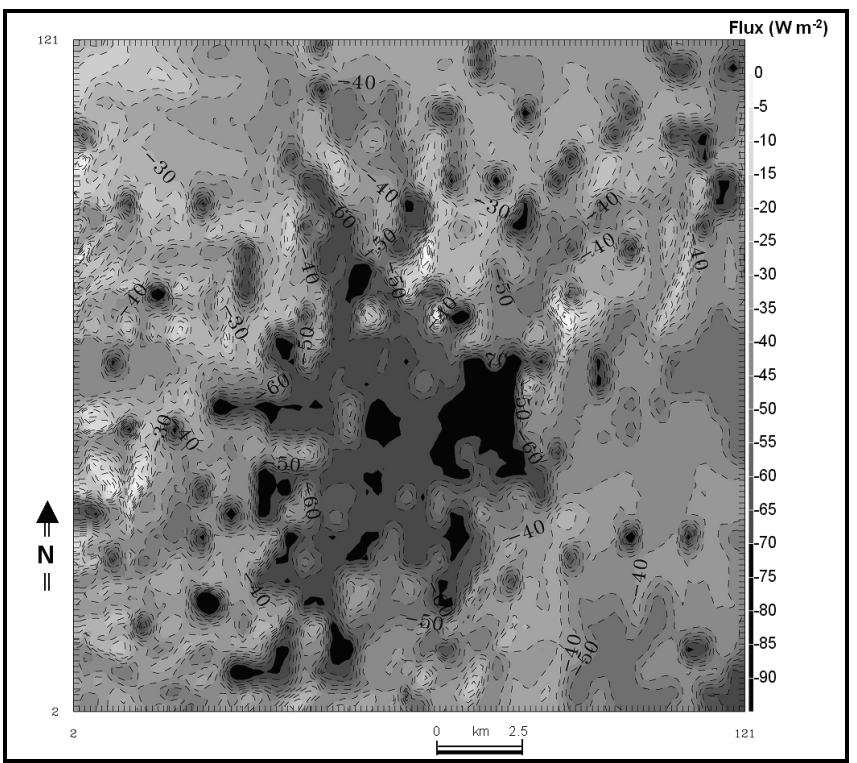

Quelques zones au sein de la ville font exception (taches claires) et correspondent aux zones plus végétalisées (parcs). En-dehors de la ville, dès qu'une maille possède une quantité non négligeable d'espace urbain, elle se singularise, ce qui fait ressortir l'emplacement des villages environnants (taches foncées à la campagne). Finalement, ce type de carte prouve que la ville apparaît effectivement la nuit comme une entité spatiale nettement différente de la campagne environnante en termes de processus. 
Cette remarque nous amène à considérer ce qui se passe à la campagne, et ceci pour deux raisons essentielles : $i$ ) la simulation urbaine ne peut être réussie que si le modèle fonctionne bien aussi en zone rurale. En effet, il existe une interaction entre l'atmosphère urbaine et l'atmosphère rurale via les processus d'advection. Ces phénomènes sont d'autant plus marqués que la ville est petite; ii) Les résultats urbains sont, dans la plupart des études, comparés à ceux obtenus dans un site rural dit "de référence ». Il faut donc que le modèle soit aussi réaliste en zone rurale qu'en zone urbaine de façon à permettre cette comparaison qui fera ressortir les caractéristiques urbaines. Malheureusement, le choix de la référence rurale se pose d'autant plus qu'il existe une très grande variété de configurations rurales (tout comme il existe une grande variété de quartiers urbains). C'est pourquoi nous allons comparer certains termes du bilan d'énergie de la ville avec ceux de quatre sites ruraux, plus ou moins éloignés de l'agglomération et placés dans des contextes différents: il s'agit des sites d'Entzheim, Hegeney, CRR et Geispolsheim (voir la carte de localisation en figure 3).

Pour le flux de chaleur latente (en haut de la figure 9), la différence est flagrante entre la maille purement urbaine (où le flux est extrêmement faible), et les autres. La différence entre le site rural d'Hegeney et la ville atteint jusqu'à $400 \mathrm{~W} \mathrm{~m}^{-2}$. A l'inverse, le flux de chaleur sensible (figure 9, au milieu) est bien plus fort en ville qu'à la campagne. Mais les écarts sont un peu moins marqués que pour le flux de chaleur latente (la différence est de l'ordre de 300 $\mathrm{W} \mathrm{m}^{-2}$ ).

Figure 9 : Comparaison de quelques composantes du bilan d'énergie simulées pour les mailles "Ville, Entzheim, Hegeney, CRR, Geispolsheim" du 13 au 17 août 2002. Flux de chaleur latente (en haut), flux de chaleur sensible (milieu) et flux de chaleur dans le sol (en bas). Résultats issus du modèle $\mathrm{N}^{\circ} 2$. Comparison of some energy balance components simulated for the sites " town, Entzheim, Hegeney, CRR, Geispolsheim " from august 13 to august 17, 2002. Latent heat flux (top), sensible heat flux (middle), ground heat flux (bottom). The results are obtained with model $n^{\circ} 2$.

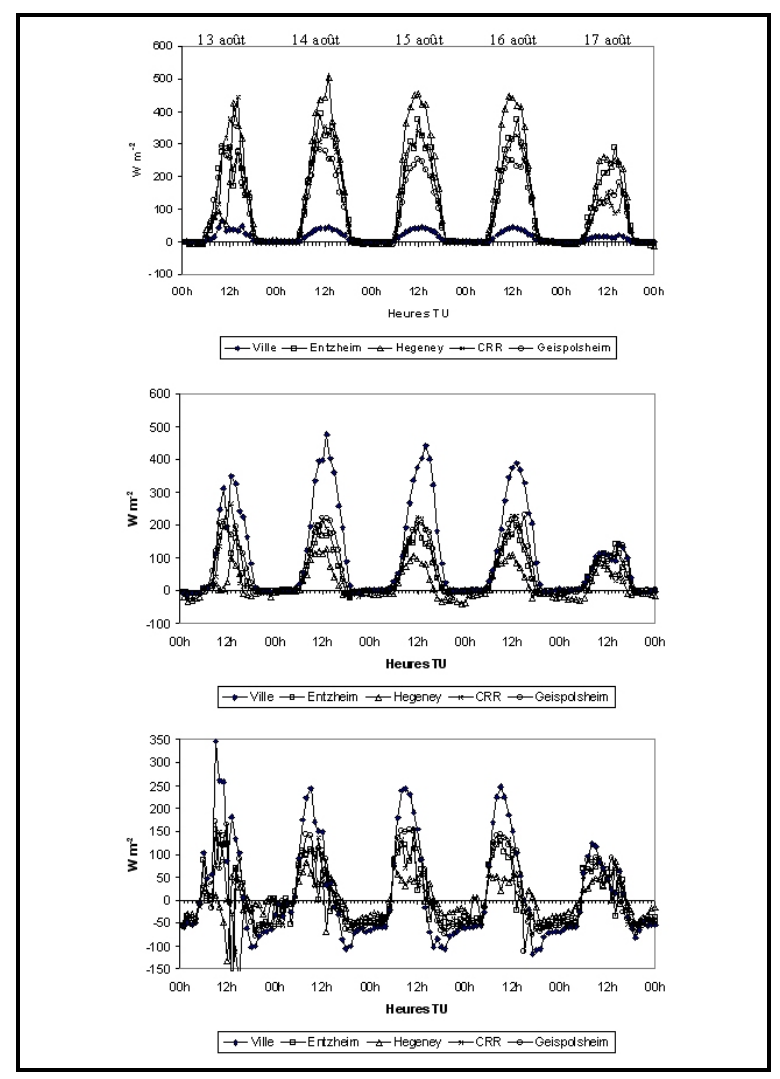

Bien entendu, le flux prépondérant change en fonction du type de maille : plus la maille est rurale, plus le flux de chaleur latente prend de l'importance, jusqu'à devenir prépondérant. Pour le flux de chaleur dans le sol (figure 9, en bas), les écarts entre la ville et la campagne sont assez faibles (seulement $200 \mathrm{~W} \mathrm{~m}^{-2}$ d'écart au grand maximum). C'est en matinée que la différence est la plus notable : la ville a tendance à stocker plus d'énergie que la campagne. La nuit, lors de la phase de destockage, les différences sont moindres $\left(30 \mathrm{~W} \mathrm{~m}^{-2}\right.$ d'écart 
seulement), mais c'est à la ville que ce terme est le plus négatif, ce qui rejoint les remarques faites à propos des figures 6 et 8 .

A y regarder de plus près, on constate une gradation des valeurs en fonction du degré d'urbanisation de la maille. En effet, même si les quatre sites sont assimilés à des sites ruraux, il faut rappeler que les résultats sont issus de mailles de $4 \mathrm{~km}^{2}$ (en provenance du modèle $\mathrm{N}^{\circ} 2$ ). Celles-ci possèdent forcément une végétation disparate et ont toutes les chances d'englober des constructions en plus ou moins grande proportion. L'introduction de ces portions urbaines induit des différences entre sites qui se traduisent sur la répartition des composantes du bilan d'énergie. Comme cela a déjà été souligné précédemment, le flux de chaleur latente notamment est le plus fort dans la maille la plus rurale (Hegeney), où il atteint facilement $450 \mathrm{~W} \mathrm{~m}^{-2}$, alors que c'est dans cette maille que le flux de chaleur sensible (100 $\left.\mathrm{W} \mathrm{m}^{-2}\right)$ et le flux de chaleur dans le sol $\left(50 \mathrm{~W} \mathrm{~m}^{-2}\right)$ sont les plus faibles. Dans la maille dite " Geispolsheim », qui est le site le plus urbanisé, le flux de chaleur latente est du même ordre de grandeur que le flux de chaleur sensible $\left(250 \mathrm{~W} \mathrm{~m}^{-2}\right)$ et le flux de chaleur dans le sol augmente (entre 100 et $150 \mathrm{~W} \mathrm{~m}^{-2}$ ). Le rapport de Bowen (figure 10) est tout à fait révélateur à ce sujet, puisque plus la maille est rurale, plus il est faible (le rapport de Bowen est le rapport entre le flux de chaleur sensible et latente). Par ailleurs, sa cinétique diurne semble être différente en fonction du degré d'urbanisation de la maille : il est plutôt fort en début de journée et s'affaiblit ensuite pour les mailles très rurales (type Hegeney), alors que pour les mailles plus urbaines (type Geispolsheim), il est plutôt faible le matin et augmente l'aprèsmidi.

Figure 10: Rapport de Bowen calculé d'après les données simulées pour les mailles "Entzheim, Hegeney, CRR, Geispolsheim" du 13 au 17 août 2002. Bowen ratio computed with the simulated data of the "Entzheim, Hegeney, CRR, Geispolsheim » sites from august 13 to august 17, 2002.

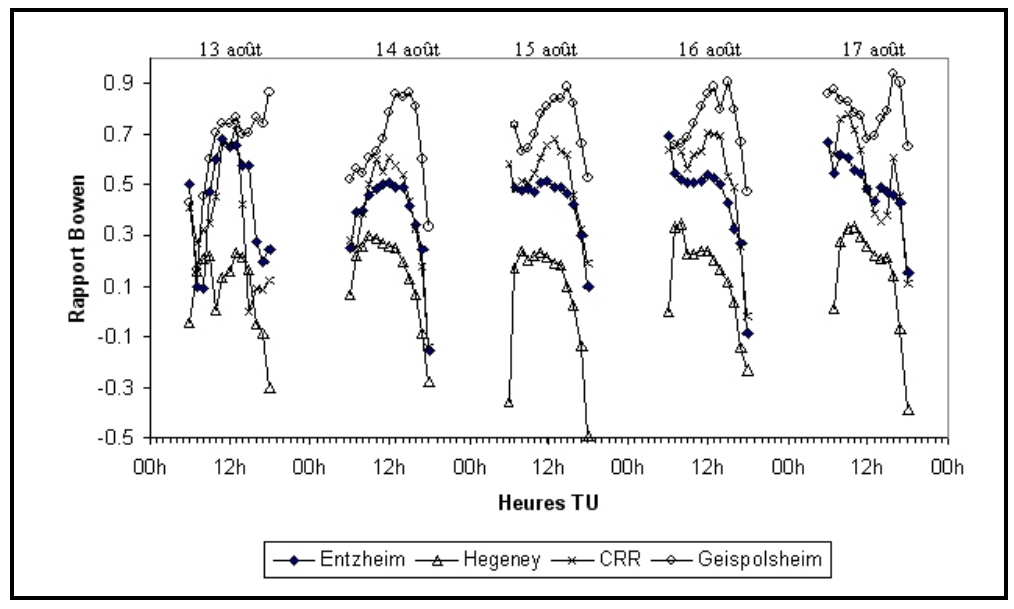

En analysant les valeurs obtenues par les différents termes du bilan d'énergie au cours de la nuit, il apparaît que les seuls échanges significatifs y ont lieu sous forme de rayonnement infrarouge (qui sont inclus dans le RN) et de flux de chaleur dans le sol (le flux de chaleur sensible et latente sont quasiment nuls et le rayonnement solaire n'existe pas). En conséquence, le RN et le flux de chaleur dans le sol se compensent alors l'un l'autre (ce qui est une particularité nettement visible sur la figure 6). La chaleur puisée dans les objets se transforme en rayonnement infrarouge terrestre.

\section{Les températures et les pressions partielles en vapeur d'eau}

Cette partie aborde l'analyse de paramètres météorologiques plus classiques que sont notamment les températures et les pressions partielles en vapeur d'eau. Etant donné les différences significatives qui existent dans les termes du bilan d'énergie entre la ville et la campagne, on peut supposer que ces différences se font ressentir aussi sur les températures et l'humidité des basses couches de l'atmosphère. 
Selon les données qui ont été mesurées au centre de Strasbourg lors de ces belles journées d'été, la température de l'air n'a cessé d'augmenter en ville sous l'effet de l'accumulation de la chaleur. Ceci se constate aussi bien au niveau du maximum (qui passe de $24^{\circ}$ à $29^{\circ} \mathrm{C}$ ) qu'au niveau du minimum (qui passe de $16,4^{\circ}$ à $17,9^{\circ}$ ). De même, l'air s'enrichit en vapeur d'eau grâce à l'importante évapotranspiration campagnarde (la pression partielle passe de $14 \mathrm{hPa}$ à $19 \mathrm{hPa}$ sur toute la période).

Pour montrer la spécificité du climat urbain, il est classique de calculer les écarts de températures ou de pression partielles en vapeur d'eau entre la ville et la campagne. Pour ce faire, c'est le site d'Entzheim qui a été choisi pour représenter la campagne. La ville sera représentée par sa maille centrale (ou la place Kleber). Comme il n'existe pas de différence d'altitude notable entre ces sites, les données n'ont pas besoin d'être corrigées (la ville est à $140 \mathrm{~m}$, Entzheim à $150 \mathrm{~m}$ ). Les écarts de températures et de vapeur d'eau vont être calculés d'après les observations (comme référence) et avec la simulation afin de pouvoir être comparés. Pour respecter les règles en la matière, notons que les variables observées et simulées sont prises au même niveau par rapport au sol, puisque le modèle calcule les températures et l'humidité à $2 \mathrm{~m}$ dans la rue, ce qui correspond aussi aux mesures.

L'écart de températures calculé entre la ville et Entzheim ne révèle rien de bien original (figure 11). Cette figure est même plutôt représentative de ce qui peut être obtenu expérimentalement en climatologie urbaine, ce qui prouve la qualité de la simulation. Ces résultats démontrent les progrès qui ont été accomplis en matière de modélisation urbaine grâce à ces nouveaux schémas de surface.

Figure 11: Différences entre les températures de l'air en ville et à la campagne (Entzheim) du 13 au 17 août 2002, issues de la simulation (Delta_T) et de la mesure (Delta_T mes). Air temperature differences between the town and the rural sites (Entzheim) from august 13 to august 17, 2002. Simulated (Delta_T) and measured (Delta_T mes).

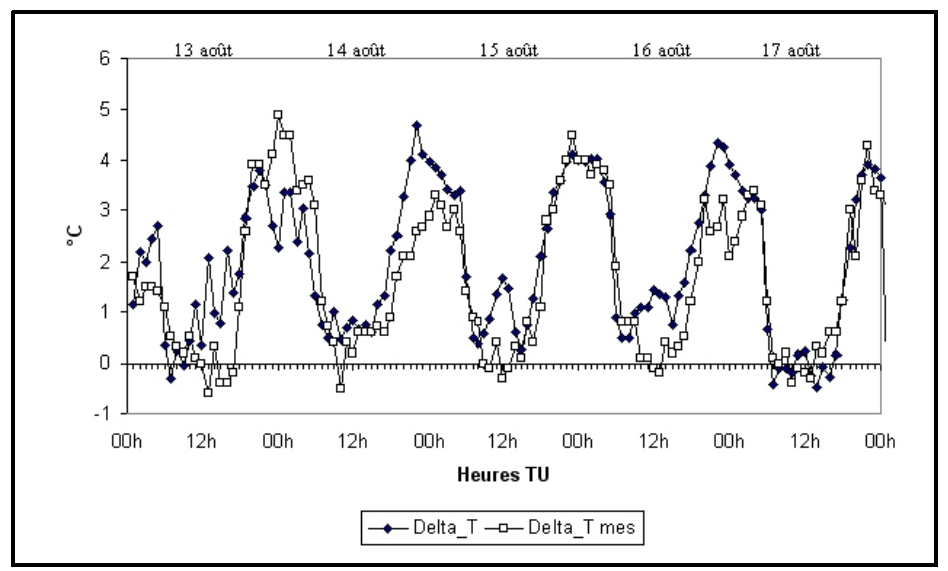

On y observe une cinétique classique, avec un îlot de chaleur urbain la nuit (l'écart est positif ce qui signifie que la ville est plus chaude que la campagne d'environ $+4^{\circ} \mathrm{C}$ ) et une isothermie diurne (l'écart devient pratiquement nul le jour). En journée, la température de l'air en ville est donc quasiment équivalente à celle de la campagne en raison du brassage vertical et de l'advection qui effacent les différences. Pendant la nuit, en revanche, l'îlot de chaleur urbain se développe pour atteindre son intensité maximale vers $23 \mathrm{~h}$. Ces résultats confirment en tout point les observations, aussi bien du point de vue du timing que de l'intensité. Evidemment, d'inévitables écarts peuvent apparaître à certains moments de la journée. Ces derniers peuvent être expliqués par le rôle local de l'advection (donc du vent), qui est un processus parfaitement aléatoire. Car n'oublions pas que la température est une variable d'équilibre entre les processus locaux et advectifs (une ventilation plus ou moins importante pourra faire varier la température de manière significative). Ainsi, les transferts de chaleur qui ont été mis en équation dans les schémas de surface de Méso-NH (à savoir TEB pour la ville et ISBA pour la campagne) sont capables d'induire des vitesses de 
refroidissement de l'air très différentes en début de nuit en fonction du milieu. C'est la raison même de leur existence dans le modèle qui se justifie ainsi.

L'îlot de chaleur urbain n'est pas un phénomène a priori homogène au niveau spatial. Il dépend, entre autre, du degré d'urbanisation de la maille, de la position de celle-ci dans la ville (configuration spatiale) et de l'advection (comme nous venons de l'évoquer). Seule une carte de l'îlot de chaleur urbain est capable de révéler ce comportement. C'est ce que confirme la figure 12, qui représente une carte de l'îlot de chaleur urbain pour les températures modélisées à $2 \mathrm{~m}$ du sol. On y distingue des quartiers où les différences de températures par rapport à la campagne sont plus ou moins prononcées, et surtout des îlots de zones fraîches au sein de la ville, notamment à l'emplacement des parcs !

Figure 12: Carte des températures de l'air (en Kelvin) à $2 \mathrm{~m}$ du sol, pour une résolution de $250 \mathrm{~m}$ (modèle $\mathrm{N}^{\circ} 3$ ) le 16 août 2002 à 23h. Map of $2 \mathrm{~m}$ above ground air temperatures (in Kelvin) of august 16, 2002 at 23 UTC. The resolution is $250 \mathrm{~m}$ (model $n^{\circ} 3$ ).

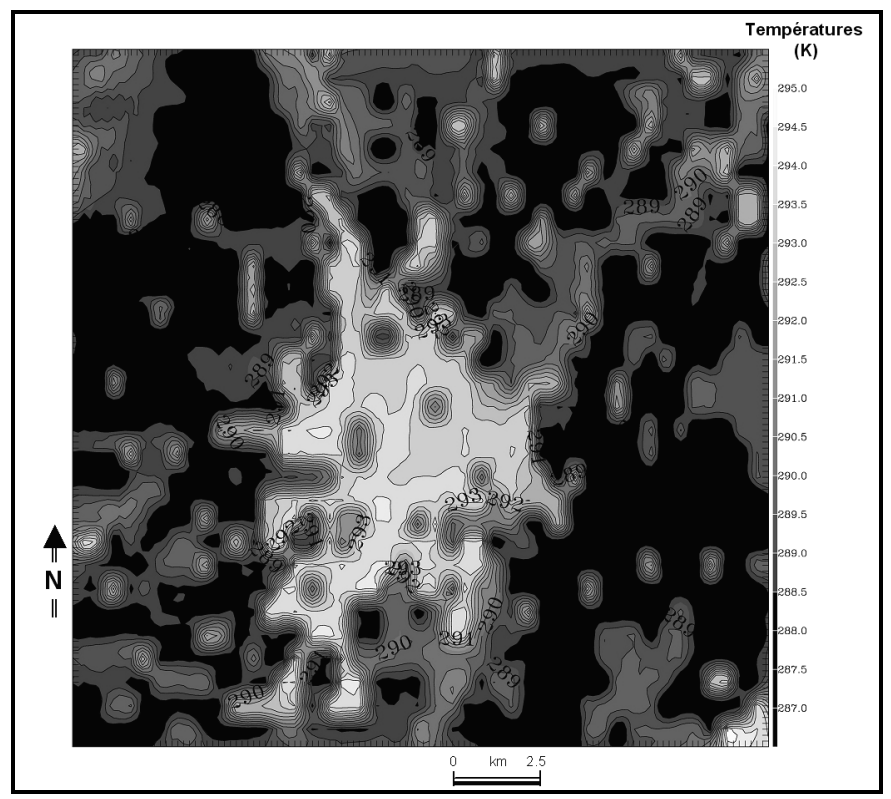

L'écart de pression partielle en vapeur d'eau entre la ville et la campagne suit aussi un cycle journalier caractéristique (figure 13). Dans ce cycle, l'air en ville est plus sec que celui de la campagne en journée (jusqu'à -2 ou $-3 \mathrm{hPa}$ de différence), ce qui paraît normal quand on connaît la faiblesse du flux de chaleur latente dans ce milieu et son importance à la campagne. Cette différence a tendance à s'atténuer dans l'après-midi en raison du brassage turbulent qui se met en place. Au cours de la nuit, la situation s'inverse : l'air en ville reste plus humide que celui de la campagne, notamment entre minuit et le lever du soleil (de +1 à $+2 \mathrm{hPa}$ ).

Figure 13: Différences entre les pressions partielles en vapeur d'eau en ville et à la campagne (Entzheim) du 13 au 17 août 2002, issues de la simulation (Delta_e) et de la mesure (Delta_e mes). Differences between the vapor pressure of the town and the rural sites (Entzheim) from august 13 to august 17, 2002. Simulated (Delta e) and measured (Delta_e mes).

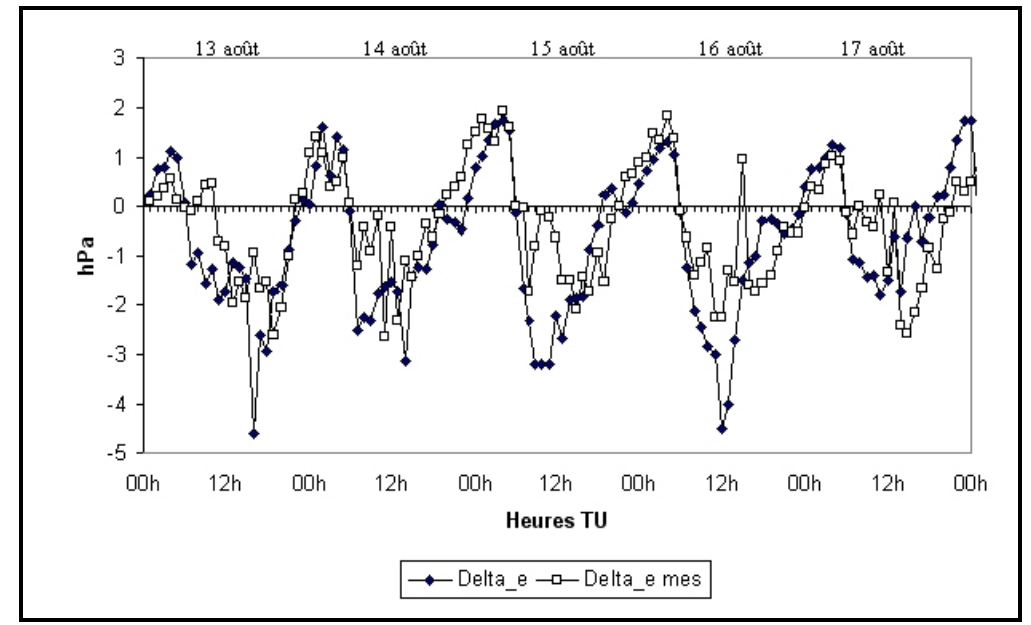


En raison de la chute importante des températures à la campagne, la capacité hygrométrique de l'air y diminue, et une partie de la vapeur peut s'y déposer sous forme de rosée. Dès le lever du soleil, la ville redevient brusquement plus sèche, puisque l'air à la campagne s'y humidifie rapidement avec la reprise de l'évapotranspiration.

Tous les résultats précédents ont été établis pour la couche d'air qui se situe sous le niveau moyen des toits, à savoir au sein de canopée urbaine et à $2 \mathrm{~m}$ du sol. Pour mettre en évidence l'effet urbain dans la couche supérieure (la CLU), il faut étudier des profils verticaux ou réaliser des coupes verticales, ce qui est extrêmement facile à faire avec un modèle. Pour faire ressortir au mieux l'influence de la ville sur des profils verticaux, il vaut mieux, une fois de plus, adopter un raisonnement par comparaison. En admettant que la campagne soit la référence, il est possible de calculer des différences entre les valeurs obtenues à différents niveaux au-dessus de la ville et de la campagne. En réalisant cette opération heure par heure et pour chaque altitude, il est possible de construire des graphiques qui retracent l'évolution de ces différences en fonction du temps et de l'altitude. Mais, une fois de plus, se pose le problème du choix de la référence rurale. Le site a été choisi de sorte à ne pas se trouver trop loin de l'agglomération, et surtout de manière à se trouver à l'amont de la ville par rapport à la direction du vent dans les basses couches. En effet, il s'agit d'éviter à tout prix qu'il se retrouve dans la trajectoire du panache urbain. Ce site est identifiable sur la figure 4 à l'aide du toponyme «profil_l».

Ce travail a été réalisé pour les températures, car c'est la variable la plus emblématique du climat urbain. D'après les résultats (figure 14), l'air au-dessus de la ville est plus chaud que celui de la campagne en journée (le delta $\mathrm{T}$ est positif en général). La figure révèle l'existence d'un panache d'air chaud (de $+0.5^{\circ} \mathrm{C}$ environ) qui s'étend verticalement au moins sur $500 \mathrm{~m}$ de haut et dont les analyses montrent qu'il se déforme horizontalement sous le vent de la ville sur des dizaines de kilomètres dans le sens du vent.

Figure 14: Différence de température vertical simulée (delta $\mathrm{T}$, en ${ }^{\circ} \mathrm{C}$ ) entre la ville et la campagne (profil_1) pour les journées du 14 au 17 août 2002 . Hauteur par rapport au sol en ordonnée et temps (en heure TU) en abscisse. Simulated vertical temperature differences (delta $T$ in Celsius) between the town and the rural site (profil_1) from august 14 to august 17, 2002. Height above ground on the vertical axis and time (UTC) on the horizontal axis.

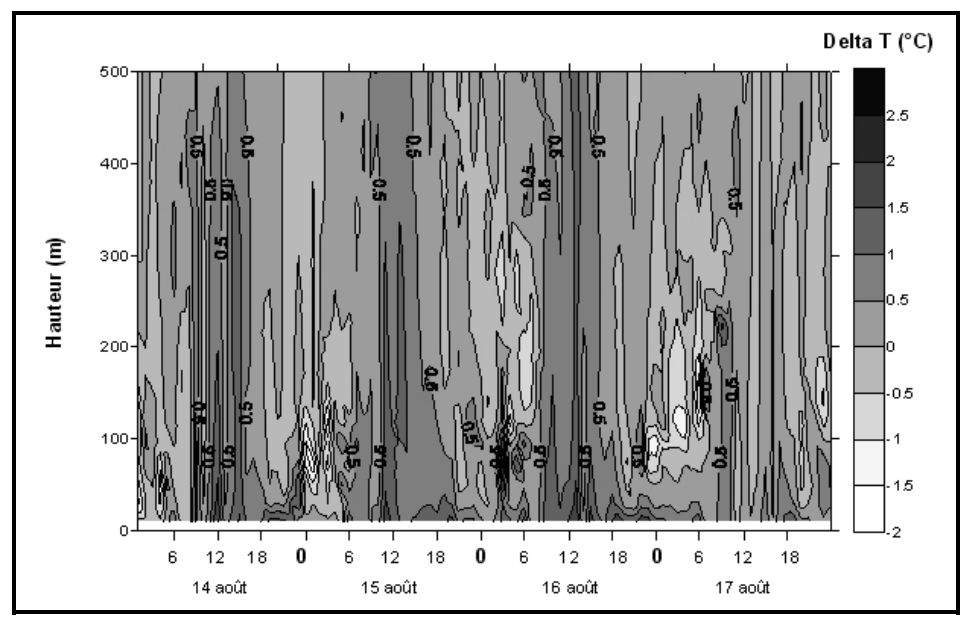

Ce panache chaud disparaît systématiquement le soir, lorsque la turbulence diminue. Il ne subsiste plus alors qu'une fine couche chaude à proximité de la surface des toits. Cet îlot de chaleur ne possède pas une grande extension verticale et cette couche est d'ailleurs d'épaisseur très variable (parfois elle n'existe même pas sous l'effet de l'advection). Audessus de cette pellicule d'air chaud, s'étend une couche où les températures sont plus fraîches qu'à la campagne (le delta $\mathrm{T}$ peut y être négatif de $-1,5^{\circ} \mathrm{C}$ ). Le sommet de cette anomalie froide marque la limite supérieure de la CLU. L'effet urbain sur les températures se manifeste donc de la façon suivante à Strasbourg : en journée, les profils de température sont quasiment isothermes (à la ville et la campagne) en raison de l'intense brassage turbulent. Néanmoins, les températures de l'air à la verticale de la ville sont un peu plus chaudes qu'à la 
campagne, ce qui marque la présence d'un panache chaud. En début de soirée, l'air près du sol se refroidit plus vite à la campagne qu'à la ville, et l'îlot de chaleur fait son apparition. En conséquence, l'inversion de surface est bien établie en zone rurale, alors qu'en ville, le profil reste isotherme sur une certaine épaisseur (du fait d'une certaine turbulence). La comparaison de ces profils thermiques met en évidence une anomalie d'air froid qui surmonte l'îlot de chaleur. Cette situation perdure plus ou moins bien durant la nuit en fonction de l'advection. Avec le lever du soleil, le réchauffement de la surface du sol provoque l'instabilité de l'air, ce qui détruit l'inversion thermique rurale par le bas. La turbulence assure l'homogénéisation verticale des températures et un nouveau cycle recommence.

\section{Conclusion}

Grâce aux schémas de surface qui se diversifient de plus en plus et qui sont maintenant capables de traiter des types d'occupation du sol spécifiques (comme la ville), les modèles météorologiques gagnent en réalisme et en fiabilité pour simuler le comportement des très basses couches de l'atmosphère. Ce sont les schémas de surface, comme TEB, qui sont chargés de simuler les interactions ville/atmosphère en tenant compte de la spécificité de la géométrie, des matériaux et des revêtements urbains. Ce sont ces contraintes qui confèrent sa particularité au climat urbain, dont l'extension se traduit par la mise en place d'un dôme ou d'un panache, dans lequel les propriétés de l'atmosphère se singularisent par rapport à la campagne avoisinante. Parmi ces singularités, le phénomène d'îlot de chaleur est l'un des plus étudié.

Les résultats de simulation présentés dans cet article permettent de décortiquer les mécanismes qui sont à l'œuvre, aussi bien au niveau radiatif, qu'au niveau des autres flux de chaleur. Ils montrent que des différences notables existent par rapport à la campagne, dont les répercussions se font sentir sur la température et la vapeur d'eau. Par ailleurs, les simulations ont l'avantage d'offrir une couverture spatiale exhaustive de la ville, ce qui permet d'obtenir des informations précieuses sur l'extension du phénomène (aussi bien verticalement qu'horizontalement). Les simulations montrent que la ville n'est pas un bloc homogène mais que de subtiles différences entre quartiers y modifient l'équilibre des flux. Les parcs urbains notamment sont de véritables îlots, nettement identifiables. Cette simulation confirme également la faible extension verticale de l'îlot de chaleur qui ne s'étend que sur quelques dizaines de mètres de haut, et qui est surmonté par une anomalie froide. Par ailleurs, l'influence de l'advection nocturne ressort nettement, surtout au-dessus des toits où elle réussit parfois à balayer complètement l'effet d'îlot de chaleur et à moduler l'épaisseur de la CLU. Enfin, un autre phénomène a été nettement mis en évidence : l'existence d'un panache urbain chaud, bien développé en journée, qui se déforme sous l'effet de l'advection dans le sens du vent et qui s'étend loin sous le vent de la ville. La richesse et la qualité de ces nouveaux résultats confirment les énormes progrès qui ont été réalisés par les modèles au cours de ces dernières années. Ils peuvent servir comme outil de recherche fondamentale, pour la capacité qu'ils ont à révéler les processus à l'œuvre, ou comme aide à la décision, ce qui les rendra bientôt indispensables en matière d'aménagement urbain.

\section{Bibliographie}

DUPONT S. et MESTAYER P. G., 2006 : Parameterization of the urban energy budget with the submesoscale soil model. Journal of Applied Meteorology and Climatology, 45, 17441765. 
HAMDI R. et SCHAYES G., 2007 : Validation of Martilli's urban boundary layer scheme with measurements from two mid-latitude European cities. Atmospheric Chemistry and Physics, 7, 4513-4526.

HEINO R., 1979 : Urban effect on climatic elements in Finland. Geophysica 15, 171-188.

KASTENDEUCH P. P. et NAJJAR G., 2009a: Simulation de l'îlot de chaleur urbain à Strasbourg. $5^{\text {ème }}$ réunion des utilisateurs de Méso-NH, 12-13 octobre 2009, Météo-France (Toulouse), mesonh.aero.obs-mip.fr/mesonh/dir_meetings/2009/presentations.html.

KASTENDEUCH P. P. et NAJJAR G., 2009b : Simulation of radiative transfers in urbanised areas. Solar Energy, 83, 333-341.

KASTENDEUCH P. P., NAJJAR G. et RINGENBACH N., 2006 : Modélisation du bilan radiatif et d'énergie d'un canyon urbain à Strasbourg, Climatologie, 3, 25-42.

KASTENDEUCH P. P., LACARRERE P., NAJJAR G., NOILHAN J., GASSMANN F. et PAUL P., 2000: Mesoscale simulations of thermodynamic fluxes over complex terrain. International Journal of Climatology, 20, 1249-1264.

LANDSBERG H.E., 1981 : The Urban Climate. Academic Press, New York, 285 pages.

MARTILLI A., CLAPPIER A. et ROTACH M. W., 2002: An urban surface exchange parameterisation for messocale models. Boundary-Layer Meteorology. Meteorology, 104, 261-304.

MASSON V. 2000 : A physically-based scheme for the urban energy budget in atmospheric models. Boundary Layer Meteorology, 94, 357-397.

NAJJAR G., KASTENDEUCH P. P., STOLL M. P., COLIN J. R., NERRY F., RINGENBACH N., BERNARD J., DE HATTEN A., LUHAHE R. et VIVILLE D., 2004 : Le projet RECLUS, télédétection, rayonnement et bilan d'énergie en climatologie urbaine à Strasbourg. La Météorologie, 46, 44-50.

NOILHAN J. et MAHFOUF J.-F., 1996 : The ISBA land surface parameterization scheme. Global and Planetary Change, 13, 145-159.

NOILHAN J. et PLANTON S., 1989 : A simple parameterization of land surface processes for meteorological models. Monthly Weather Review, 117, 536-549.

OKE T.R., 1987 : Boundary Layer Climates (second ed.). Methuen, London, 435 pages.

OTTE T., LACSER A., DUPONT S. et CHING J. K. S., 2004 : Implementation of an Urban Canopy Parameterization in a Mesoscale Meteorological Model. Journal of applied Meteorology, 43, 1648-1665.

ZHANG H., N., SATO T., IZUMI K., HANAKI T. et ARAMAKI, 2008 : Modified RAMSUrban Canopy Model for Heat Island Simulation in Chongqing, China. Journal of applied Meteorology and climatology, 47, 509-524. 
ㄷํ르 